\title{
Evaluation of lectin pathway activity and mannan-binding lectin levels in the course of pregnancy complicated by diabetes type 1, based on the genetic background
}

\author{
Magdalena Pertyńska-Marczewska¹, Maciej Cedzyński², Anna Świerzko², \\ Agnieszka Szala ${ }^{2}$, Małgorzata Sobczak ${ }^{3}$, Katarzyna Cypryk ${ }^{4}$ and Jan Wilczyński ${ }^{1}$ \\ ${ }^{1}$ Department of Fetal-Maternal Medicine and Gynecology, Research Institute Polish Mother's Memorial Hospital, Łódź, \\ Poland \\ ${ }^{2}$ Laboratory of Immunobiology of Infections, Institute of Medical Biology, Polish Academy of Sciences, Lódź, Poland \\ ${ }^{3}$ Specialized Outpatient Clinic, Department of Fetal-Maternal Medicine and Gynecology, Research Institute Polish \\ Mother's Memorial Hospital, Łódź, Poland \\ ${ }^{4}$ Department of Diabetology and Metabolic Diseases, Medical University of Łódź, Łódź, Poland
}

Received: 2008.06.09, Accepted: 2008.11.10, Published online: 2009.06.11

(C) L. Hirszfeld Institute of Immunology and Experimental Therapy, Wrocław, Poland 2009

\begin{abstract}
Introduction: There are numerous indications that either mannan-binding lectin (MBL) deficiency or its excessive activity are associated with adverse pregnancy outcomes. High MBL concentrations and corresponding $M B L 2$ genotypes were shown to be associated with microvascular complications in type 1 diabetes. The aim of this study was to evaluate levels of MBL and MBL-dependent activity of the lectin pathway (LP) of complement in the course of pregnancy in diabetic mothers, based on genetic background.

Materials and Methods: These parameters were determined in samples from healthy non-pregnant (control), diabetic non-pregnant, healthy pregnant, and pregnant diabetic women.

Results: No significant differences in median MBL levels or LP activities were found in any study group compared to the control. However, statistically significant differences in MBL levels were noted during pregnancy between the 1st and 3rd trimesters in both healthy controls and pregnant diabetics. With regard to LP values, similar trends were evident, but statistically significant results were obtained only in the healthy pregnant group. When data analysis was confined to patients carrying the A/A (wild-type) MBL2 genotype, an increase in MBL level during pregnancy (in both healthy and diabetic pregnant women) was still observed. Similarly, LP activity increased during both healthy and diabetic pregnancies, significantly so for the former. Conclusions: Diabetes, an autoimmune disease, is a serious complication of pregnancy. Therefore, determination of MBL status might be beneficial in identifying type 1 diabetic patients who are at increased risk of developing both vascular complications and poor pregnancy outcomes.
\end{abstract}

Key words: mannan-binding lectin, lectin pathway activity, diabetes, pregnancy.

Abbreviations: MBL - mannan-binding lectin, LP - lectin pathway, SNPs - single-nucleotide polymorphisms, PD - diabetic pregnant.

Corresponding author: Magdalena Pertyńska-Marczewska, Department of Fetal-Maternal Medicine and Gynecology, Research Institute Polish Mother's Memorial Hospital, Łódź, Poland, e-mail: m_pertynska@yahoo.com

\section{INTRODUCTION}

The complement system is an important part of the innate immune system. It can be activated via three different routes: the classical pathway, the alternative pathway, and the lectin pathway (LP) (Turner 1996). The LP performs a crucial role in the immune system by recognizing pathogens through patterns of sugar moieties displayed on their cell surfaces and neutralizing them via an antibody-independent reaction cascade initiated by the binding of mannan-binding lectin (MBL) or ficolins (Kilpatrick 2007). MBL plays an important role in innate immunity by recognizing and initiating the opsonization of microorganisms (Kilpatrick 2007; 
Turner and Hamvas 2000), activating complement independently of the adaptive clonal immune system and thus presenting an innate anti-microbial defense mechanism (Thiel et al. 2002). MBL is synthesized in the liver and belongs to a family of proteins known as the collectins, a group of C-type lectins possessing a collagenlike helical domain. As a C-type lectin, this pattern recognition molecule acts by binding to D-mannose, $\mathrm{N}$ -acetyl-D-glucosamine, and L-fucose residues presented with the densities and orientations commonly found on microbial surfaces, upon which it initiates complement activation through association with serine proteases (MBL-associated serine protease-1, -2, and -3) (Dahl et al. 2001; Matsushita and Fujita 1992; Thiel et al. 1997; Turner and Hamvas 2000). When bound to microorganisms, the MBL complex mediates the activation of $\mathrm{C} 4$ and $\mathrm{C} 2$, generating the $\mathrm{C} 3$ convertase $\mathrm{C} 4 \mathrm{bC} 2 \mathrm{~b}$. The $\mathrm{C} 4 / \mathrm{C} 2$ cleaving activity of the MBL complex is shared by the $\mathrm{C} 1$ complex of the classical pathway of complement activation (Thiel et al. 2002). Emerging evidence indicates that in some situations, MBL may cause activation of the complement system and tissue injury by binding to endothelial glycosylations (Hansen et al. 2003a).

Single-nucleotide polymorphisms (SNPs) in exon 1 of the $M B L 2$ gene are associated with individual MBL serum levels as well as impaired MBL function. Individuals with the A/A wild-type genotype have higher MBL serum concentrations, whereas individuals with the $\mathrm{A} / \mathrm{O}$ or $\mathrm{O} / \mathrm{O}$ genotypes (where $\mathrm{O}$ is the collective designation of the mutant dominant alleles $\mathrm{D}, \mathrm{B}$, and C corresponding to mutations in codons 52,54 , and 57, respectively) show lower MBL serum concentrations. The presence of any of the minority alleles, when heterozygous, often results in a significant reduction in serum MBL concentration, and when homozygous, in an almost complete absence of the protein (Madsen et al. 1995). Polymorphisms in the promoter and exon 1 untranslated region $(\mathrm{H} / \mathrm{L}, \mathrm{Y} / \mathrm{X}$, and $\mathrm{P} / \mathrm{Q}$ at positions $-550,-221$, and +4 , respectively) influence the gene expression level and thus the serum protein concentration. The highest MBL levels occur in association with HYP/HYP and the lowest with LXP/LXP genotypes (Madsen et al. 1995).

Pregnancy is associated with changes in the immune system. There are indications that components of innate immunity, such as MBL, are associated with pregnancy outcome (Kilpatrick 2000), but the way in which innate immunity changes during pregnancy has not been extensively studied. There is some evidence that deficient maternal MBL concentrations could act as a risk factor for preterm birth and low birth weight (Annelss et al. 2004). However, Megia et al. (Megia et al. 2004) demonstrated that pregnant women bearing the G54D MBL allele have a greater risk of developing GDM and having heavier infants. On the other hand, high MBL concentrations as well as the corresponding $M B L 2$ genotypes were shown to be associated with microvascular complications in type 1 diabetes (Bouwman et al. 2005; Hansen et al. 2004).
In a preliminary report we demonstrated higher levels of MBL in diabetic non-pregnant than in healthy non-pregnant women (Pertynska et al. 2001). The aim of the present study was to evaluate MBL levels and the activity of the LP in the course of pregnancy in diabetic mothers based on genetic background, i.e. MBL2 gene exon 1 and promoter region SNPs.

\section{MATERIALS AND METHODS}

Fifteen pregnant (primigravidae) diabetic type 1 women (PD), patients of Diabetes Centre Research Institute Polish Mother's Memorial Hospital (RI PMMH), demonstrating good glycemic control, were first studied at 11-13 ( $\mathrm{n}=15$, group PD1) then at 34-36 $(\mathrm{n}=12$, group PD3) weeks of gestation (according to the date of the last menstrual period confirmed by ultrasound). Fourteen non-pregnant type 1 diabetic women (D) of the Outpatients Diabetes Center of the RI PMMH, 15 healthy non-diabetic nulliparous women (C), and 16 healthy pregnant (primigravidae) women (P) between 11-13 ( $\mathrm{n}=16$, group $\mathrm{P} 1)$ and again between 34-36 $(n=10$, group P3) weeks of gestation matched for age and parity were also studied. All of the patients were between 20-30 years of age (mean: 23.6 years); all the pregnant women were primigravidae. No patient had clinical signs of active infection at the time of the study and for at least 4 weeks prior to it. All the diabetic women had fasting glucose levels $<110 \mathrm{mg} / \mathrm{dl}$. Glycosylated hemoglobin (HbA1C) concentration was measured using an Integra HbA1C 150 kit manufactured by Roche. Fasting blood samples of $5 \mathrm{ml}$ were obtained from an antecubital vein between 8 and 9 AM. Serum samples were obtained from blood taken into Vacutainer tubes. Agreement of the local ethics committee as well as informed consent from all the investigated patients had been obtained.

\section{MBL level and LP activity determination}

MBL concentration in human serum and LP activity were determined essentially as described by Cedzynski et al. (Cedzynski et al. 2004). Briefly, diluted serum was incubated on mannan-coated plates and bound MBL was detected using murine anti-human MBL (clone1311; AntibodyShop, Denmark), peroxidase-conjugated rabbit anti-mouse immunoglobulin (Sigma, USA), and 2,2'-azino-bis(3-ethylbenzthiazoline-6-sulfonic) acid (Sigma, USA). The MBL-MASP-2 complex-dependent LP of complement activity (C4b deposition) was also measured by ELISA as previously described according to the method originally described by Petersen et al. (Petersen et al. 2001) in our modification (Cedzynski et al. 2004). Briefly, serum serially diluted in a high-ionic-strength buffer (Petersen et al. 2001) was incubated on mannan-coated plates. Then complement component C4 (Sigma, USA) was added and, after incubation and washing steps, the primary antibody (rabbit anti-human 
C4 serum from Sigma, USA) was added, followed by the secondary antibody (peroxidase conjugated goat anti-rabbit Ig from DAKO, Denmark). 2,2'-azino-bis(3-ethylbenz-thiazoline-6-sulfonic) acid (ABTS, Sigma, USA) was employed as the substrate for peroxidase. The serum of a healthy blood donor of HYPA/HYPA genotype containing $2000 \mathrm{ng} / \mathrm{ml}$ MBL was used as a standard for which LP activity was arbitrarily determined as 1000 $\mathrm{mU} / \mathrm{ml}$.

\section{MBL2 genotyping}

DNA was extracted from the blood samples according to the GTC method (Chomczynski 1993). Exon 1 $M B L 2$ gene was amplified by PCR (685 bp) using the primers 5' AGTCGACCCAGATTGTAGGACAGAG $3^{\prime}$ and 5' AGTTGTTGTTCTCCTGTCCAG 3' (Madsen et al. 1995). The PCR products were digested by BanI and MboII restriction enzymes and separated on a $6 \%$ polyacrylamide gel.

The $\mathrm{D}$ allele was detected by the RFLP method performed on the PCR products of $125 \mathrm{bp}$ using the primers 5' CATCAACGGCTTCCCAGGCAAAGACGCG $3^{\prime}$ and $5^{\prime}$ AGGATCCAGGCAGTTTCCTCGGAAGG 3' (Madsen et al. 1995). The PCR product was digested by MluI and HhaI restriction enzymes and electrophoresed on a $6 \%$ polyacrylamide gel in TAE buffer.

The cis-trans promoter/exon 1 untranslated region variants $\mathrm{H}, \mathrm{L}, \mathrm{X}, \mathrm{Y}, \mathrm{P}$, and $\mathrm{Q}$ relative to the structural variants $\mathrm{A}, \mathrm{B}, \mathrm{C}$, and $\mathrm{D}$ were determined by PCR using the primers 5' GGAGGCTTAGACCTATGGGGCTAGG 3' and 5' GGGACATGGTCCTCACCTTGGTG 3' (Madsen et al. 1995). The appropriate PCR products of $866 \mathrm{bp}$ were separated on Wizard columns (Promega, USA) and sequenced by the dideoxy chain termination method (Epicentre Technologies, USA) starting from the primers specific for $\mathrm{H} / \mathrm{L}$ : 5' CCAACGTAGTAAGAAATTTCC 3', X/Y: 5' GGCATAAGCCAGCTGGCAATGC 3', and P/Q: 5' GGGATGGGTCATCTATTTCTATATAGCC 3 (Madsen et al. 1995).

\section{Statistics}

The Statistica 8.0 (StatSoft Poland) software package was used for data management and statistical calculations. Results are shown as medians. For statistical evaluation the Mann-Whitney U-test or Wilcoxon test were implemented where appropriate. A p value of $<0.05$ was considered as indicative of a significant difference.

\section{RESULTS}

MBL concentrations and LP activity were determined in serum samples from 15 healthy non-pregnant (control group C), 14 non-pregnant diabetic (D), 16 healthy pregnant women in the first trimester (P1), and 15 pregnant diabetic women in the first trimester (PD1). No significant differences in median MBL level were found in any studied group (D, P1, or PD1) compared to the control (Table 1). Similarly, median LP, reflecting the ability to deposit C4b, did not differ significantly among the healthy pregnant, pregnant diabetic, or non-pregnant diabetic patients and healthy non-pregnant nulliparous controls (Table 2). Two persons in group $\mathrm{P}$, three in $\mathrm{D}$, two in $\mathrm{CD}$, and none in $\mathrm{C}$ were found to be MBL deficient (concentrations not exceeding $100 \mathrm{ng} / \mathrm{ml}$ ).

Furthermore, MBL concentrations and LP activity were determined in serum samples from 13 pregnant diabetic women in the third trimester (PD3) and 10 healthy pregnant women in the third trimester (P3). The numbers in the third trimester groups differ from the first trimester due to two miscarriages in the diabetic pregnant group and the fact that six of the healthy pregnant patients either decided to deliver in another city $(n=5)$ or did not appear for blood collection $(n=1)$. Again, no significant differences in median MBL level were found with any group studied (D, P3, or PD3) compared to the control. Similarly, median LP did not differ significantly among the healthy pregnant, pregnant diabetic, or non-pregnant diabetic patients and the

Table 1. MBL concentration $[\mathrm{ng} / \mathrm{ml}]$ in sera of healthy non-pregnant (C), non-pregnant diabetic (D), healthy pregnant $(\mathrm{P})$, and diabetic pregnant $(\mathrm{PD})$ women in the 1 st and $3 \mathrm{rd}$ trimesters

\begin{tabular}{lllrl}
\hline Group & Median & Mean & \multicolumn{1}{l}{ Range } & $\mathrm{p}$ \\
\hline C & 1807 & 2008 & $140-5819$ & \\
D & 1114 & 2067 & $21-6601$ & $0.73^{\mathrm{a}}$ \\
& & & & $0.85^{\mathrm{b}}$ \\
P1 & 2118 & 2332 & $88-5593$ & $0.65^{\mathrm{a}}$ \\
& & & & $0.69^{\mathrm{b}}$ \\
PD1 & 1542 & 2168 & $34-5895$ & $0.97^{\mathrm{a}}$ \\
P3 & 2932 & 2934 & $115-6074$ & $0.005^{\mathrm{c}}$ \\
PD3 & 1877 & 2603 & $33-6236$ & $0.003^{\mathrm{b}}$ \\
\hline
\end{tabular}

${ }^{\mathrm{a}}$ vs. C, ${ }^{\mathrm{b}}$ vs. PD1, ${ }^{\mathrm{c}}$ vs. P1

Table 2. LP activity [mU/ml] in sera of healthy non-pregnant (C), non-pregnant diabetic (D), healthy pregnant (P), and diabetic pregnant (PD) women in the 1st and 3rd trimesters

\begin{tabular}{lcccl}
\hline Group & Median & Mean & Range & $\mathrm{p}$ \\
\hline C & 591 & 643 & $96-2242$ & \\
D & 565 & 733 & $27-1972$ & $0.66^{\mathrm{a}}$ \\
& & & & $0.73^{\mathrm{b}}$ \\
P1 & 747 & 745 & $72-1373$ & $0.45^{\mathrm{a}}$ \\
& & & & $0.9^{\mathrm{b}}$ \\
PD1 & 834 & 933 & $40-3229$ & $0.51^{\mathrm{a}}$ \\
P3 & 1257 & 1049 & $38-1755$ & $0.013^{\mathrm{c}}$ \\
PD3 & 1242 & 1192 & $63-2954$ & $0.06^{\mathrm{b}}$ \\
\hline
\end{tabular}

${ }^{\mathrm{a}}$ vs. C, ${ }^{\mathrm{b}}$ vs. PD1, ${ }^{\mathrm{c}}$ vs. P1 
healthy non-pregnant nulliparous women. However, statistically significant differences in MBL levels between the first and third trimesters were noted in both the healthy pregnant ( $\mathrm{P} 1$ vs. $\mathrm{P} 3, \mathrm{p}=0.005)$ and the pregnant diabetic groups (PD1 vs. PD3, $p=0.003)($ Table 1$)$. With regard to LP values, a statistically significant difference in LP activity between the first and third trimesters was noted for the healthy pregnant group (P1 vs. $\mathrm{P} 3, \mathrm{p}=0.013)$. A similar trend was evident in the pregnant diabetic group, but it did not reach statistical significance $(\mathrm{p}=0.06)$ (Table 2$)$.

We also determined $M B L 2$ genotypes, and then serum MBL levels, and LP activities in the subjects who possessed the A/A genotype only. Similarly, we found an increase in MBL level in both healthy controls and diabetic patients during the course of pregnancy. LP activity increased significantly in the course of healthy pregnancies, but only a weak trend was apparent in the diabetic patients $(\mathrm{p}=0.18$; Table 3$)$. It should be mentioned that, as expected, the distribution of $\mathrm{O}$ variants was similar in the studied groups: among 16 healthy pregnant women, 2 were A/O heterozygotes (LYPA/LYPB and LXPA/HYPD) and 1 had the homozygous LYPB/LYPB genotype; among 13 pregnant diabetic patients, 5 had A/B genotypes (2 LYQA/LYPB, 2 LXPA/LYPB, and 1 HYPA/LYPB); 6 of 15 healthy non-pregnant women carried the B (3 HYPA/LYPB and 2 LXPA/LYPB) or C (HYPA/LYQC) variant; and among 14 diabetic non-pregnant patients, 5 had $\mathrm{A} / \mathrm{B}$ heterozygotes (2 HYPA/LYPB and 3 LXPA/LYPB) and 1 had variant LYPB/HYPD heterozygote. All totally MBL-deficient individuals (concentrations $<100 \mathrm{ng} / \mathrm{ml}$ ) had the $\mathrm{O} / \mathrm{O}$ or LXPA/O genotypes.

The glycosylated hemoglobin (HbA1C) results are shown in Table 4. As expected, significant differences were noted in the D and PD groups compared to the control. No differences were found between groups $\mathrm{D}$ and $\mathrm{PD}$, which indicates good glycemic control of the pregnant diabetic women, with medians of $7.0 \mathrm{mg} \%$ for group D, $7.0 \mathrm{mg} \%$ for PD1, and $6.4 \mathrm{mg} \%$ for PD3. The statistical analysis did not reveal any significant correlation between MBL levels or LP activities and HbA1C in the studied diabetic groups (data not shown).
The glucose levels did not differ significantly between diabetic pregnant women bearing the $\mathrm{A} / \mathrm{A}$ and $\mathrm{A} / \mathrm{B}$ genotypes. In the first trimester, median values were $183 \mathrm{mg} \%$ (genotype $\mathrm{A} / \mathrm{A}$ ) and $107 \mathrm{mg} \%$ (A/B, $\mathrm{p}=0.09)$. In the third trimester the levels were $147 \mathrm{mg} \%$ and $112 \mathrm{mg} \%$, respectively $(\mathrm{p}=0.09)$.

\section{DISCUSSION}

The serum level of MBL is genetically determined to a major extent (Husby et al. 2002), although non-genetic influences are also relevant (Sorensen et al. 2006) and it is well known that individual concentrations within the same genotype may vary significantly. Hansen et al. (Hansen et al. 2004) showed that the higher concentrations of MBL among diabetic patients were not explained by genetic predisposition because they found identical genotype distributions in patients and healthy control subjects. In early-onset diabetic patients, MBL serum concentration and activity were increased upon manifestation independently of genetic predisposition to high MBL production, indicating a possible role in the immunopathogenesis of type 1 diabetes in addition to adaptive islet autoimmunity (Bouwman et al. 2005). Tsutsumi et al. suggested that possession of the minori-

Table 4. Concentration of glycated hemoglobin (HbA1C) [\%] in sera of healthy non-pregnant $(\mathrm{C})$, non-pregnant diabetic (D), healthy pregnant $(\mathrm{P})$, and diabetic pregnant (PD) women in the course of pregnancy (1st and 3rd trimesters)

\begin{tabular}{lccl}
\hline Group & Median & Mean & \multicolumn{1}{c}{$\mathrm{p}$} \\
\hline C & 5.2 & 5.2 & \\
D & 7.0 & 7.6 & $\begin{array}{l}<0.00001^{\mathrm{a}} \\
0.25^{\mathrm{b}}\end{array}$ \\
P1 & 5.2 & 5.3 & $0.46^{\mathrm{a}}$ \\
& & & $0.00002^{\mathrm{b}}$ \\
PD1 & 7.0 & 7.0 & $<0.00001^{\mathrm{a}}$ \\
P3 & 5.2 & 5.3 & $0.44^{\mathrm{c}}$ \\
PD3 & 6.4 & 6.3 & $0.084^{\mathrm{b}}$ \\
\hline
\end{tabular}

${ }^{a}$ vs. C, ${ }^{b}$ vs. PD1, ${ }^{c}$ vs. P1

Table 3. Comparison between MBL [ng/ml] and LP [mU/ml] levels in patients with A/A genotype: healthy non-pregnant (C), non-pregnant diabetic (D), healthy pregnant (P, 1st and 3rd trimesters), and diabetic pregnant (PD, 1st and 3rd trimesters)

\begin{tabular}{|c|c|c|c|c|c|c|}
\hline \multirow[t]{2}{*}{ Group } & \multicolumn{2}{|c|}{ MBL [ng/ml] } & \multirow{2}{*}{$\begin{array}{l}\text { Statistical } \\
\text { significance }\end{array}$} & \multicolumn{2}{|c|}{$\mathrm{LP}[\mathrm{mU} / \mathrm{ml}]$} & \multirow{2}{*}{$\begin{array}{l}\text { Statistical } \\
\text { significance }\end{array}$} \\
\hline & median & mean & & median & mean & \\
\hline $\mathrm{C}$ & 2420 & 3005 & & 757 & 909 & \\
\hline $\mathrm{D}$ & 4057 & 3307 & & 1238 & 1107 & \\
\hline $\mathrm{P} 1$ & 2848 & 3324 & & 813 & 996 & \\
\hline $\mathrm{P} 3$ & 3244 & 3540 & $\mathrm{p}=0.012^{\mathrm{a}}$ & 1382 & 1272 & $\mathrm{p}=0.017^{\mathrm{a}}$ \\
\hline PD1 & 3163 & 3250 & & 1328 & 1446 & \\
\hline PD3 & 4008 & 4419 & $\mathrm{p}=0.018^{\mathrm{b}}$ & 1598 & 1773 & $\mathrm{p}=0.18^{\mathrm{b}}$ \\
\hline
\end{tabular}

${ }^{\mathrm{a}}$ vs. P1, b vs. PD1 
ty allele of the $M B L 2$ gene may be a minor risk factor for type 1 diabetes (Tsutsumi et al. 2003). In a preliminary study in 2001 we demonstrated higher levels of MBL in diabetic non-pregnant compared to healthy non-pregnant women (Pertynska et al. 2001).

Hansen et al. demonstrated that circulating MBL concentrations were significantly elevated in patients with diabetes type 1 and suggested a possible role of MBL in the pathogenesis of renovascular complications in diabetes (Hansen et al 2003a). In a study by Hovind et al. (Hovind et al. 2005), high levels of MBL early in the course of type 1 diabetes were significantly associated with a higher risk of developing persistent micro- or macroalbuminuria. This finding again suggests that complement activation initiated by MBL is likely to be a contributing factor in the pathogenesis of diabetic microangiopathy (Hovind et al. 2005). In diabetic patients, treatment with insulin could play a very important role in regard to MBL level. Hansen et al. (Hansen et al. 2003b) showed that insulin has a significant suppressive effect on circulating MBL levels in critically ill patients. To explain the difference in MBL level between healthy and diabetic patients they suggested that in patients with diabetes type 1 , treatment with injectable insulin preparations is associated with systemic hyperinsulinemia and hepatic portal hypoinsulinemia. As MBL is a protein synthesized mainly in the liver, in such a case hepatic MBL expression may be chronically unregulated in these patients because of the low portal insulin concentration (Hansen et al. 2003a). In the present study we did not notice any statistically significant difference in the levels of MBL or LP activity between the corresponding groups studied. This, however, could be due to the good metabolic control of our diabetic and pregnant diabetic patients (median HbA1C for groups D: $7.0 \mathrm{mg} \%$, PD1: $7.0 \mathrm{mg} \%$, PD3: $6.4 \mathrm{mg} \%$ ). Although we noted increasing levels of MBL in the course of diabetic pregnancy, only two of patients had retinopathy, in both cases as a preexisting condition.

Pregnancy is associated with changes in the immune system. There are indications that components of innate immunity, such as MBL, may influence pregnancy outcome. Although this would suggest that pregnancy also involves adaptations in innate immunity, there are only a few studies addressing this issue. In a Dutch study, MBL concentrations increased by up to $140 \%$ during pregnancy. This increase was already present at the 12th week of pregnancy and was most pronounced in the high-production A/A genotype. However, the effect of the promoter polymorphisms could also be observed (van de Geijn et al. 2007). On the other hand, Kilpatrick showed that this increase in MBL concentration may not always occur in the first trimester (Kilpatrick 2000). He noted that in eight women with a history of recurrent spontaneous miscarriage, no increase in MBL could be observed during the first trimester of pregnancy. This might suggest that a rise in MBL level in early pregnancy is important for proper development. Low maternal serum MBL levels showed a negative impact on preg- nancy outcome in women with unexplained recurrent spontaneous abortion. Patients with MBL levels of $\leq 100$ $\mathrm{ng} / \mathrm{ml}$ had a higher abortion rate than patients with normal MBL concentrations $(\mathrm{p}<0.05)$. The median birth weight of children who were born at term to women with recurrent spontaneous abortion was $287 \mathrm{~g}$ less in the women with MBL levels of $\leq 100 \mathrm{ng} / \mathrm{ml}$ than in the women with normal concentrations $(\mathrm{p}=0.04)$ (Kruse et al. 2002).

In our preliminary report (Pertynska et al. 2001) we did not notice any significant increase in MBL concentration in early pregnancy. Similarly, in the present study we did not observe any significant rise in MBL level or LP activity among women between 11 and 13 weeks of pregnancy compared to the corresponding control groups of non-pregnant women. However, we showed a significant increase in MBL levels in a healthy and, for the first time, a diabetic pregnant group in the course of pregnancy (when the values determined during the first and third trimesters were compared). Moreover, we showed that LP activity, reflecting the ability to deposit C4b, increased in the course of healthy pregnancy and a similar trend was noticed in the diabetic pregnant group $(p=0.06)$. When data from patients carrying A/A genotypes were compared to avoid statistical noise caused by MBL deficiency, an increase in MBL level in the healthy and diabetic pregnant groups in the course of pregnancy was again observed. Similarly, LP activity increased significantly in the course of healthy, but not diabetic pregnancy.

Megia et al. showed that the mean birth weight of babies of GDM mothers carrying the codon 54 mutation (B allele) was significantly higher than in a group of women homozygous for the wild-type gene (3326.4 \pm 546.9 vs. $3087.5 \pm 395.5 \mathrm{~g}, \mathrm{p}<0.05$ ) (Megia et al. 2004). We observed a similar trend in type 1 diabetic mothers $(4020 \pm 336.1$ vs. $3882.9 \pm 696.9 \mathrm{~g})$. The difference was not significant; however, it should be remembered that the number of samples studied was very small. Megia et al. analyzed the basal glucose levels in the tolerance test in the GDM group. Women bearing the mutated allele had higher mean glucose levels than those homozygous for the wild-type gene (Megia et al. 2004). We looked at the glucose levels obtained at the day of blood collection for the purposes of our study in the diabetic pregnant women. We did not find any statistical difference between women bearing the $\mathrm{A} / \mathrm{A}$ and $\mathrm{A} / \mathrm{B}$ genotypes.

There is no clear evidence concerning the purpose of increased MBL levels in pregnancy. It is well known that pregnancy is a state of powerful immune suppression, which allows the fetus to survive despite being a potential target for the maternal immune system. Multiple overlapping mechanisms exist to prevent maternal allogenic immune responses towards the fetus while maintaining the capacity to mount a defense against infectious organisms (Koch and Platt 2003). Since adaptive immunity declines during pregnancy, the mother is more susceptible to infections, both viral and (intracellular) bacterial, such as Neisseria and Mycoplasma (Beagley 
and Gockel 2003). Another factor that might contribute to increased susceptibility to viral infection is progesterone (Kaushic et al. 2003). One could hypothesize that since the balance of Th1/Th2 is crucial for pregnancy development and term delivery, then the increasing level of MBL in the course of pregnancy may be necessary to compensate for reduced T-cell function during the state of reduced adaptive immunity (van de Geijn et al. 2007; Raghupathy 1997; Szekeres-Bartho et al. 1997).

Recently, van de Geijn et al. (van de Geijn et al. 2008) found an association between maternal high MBL level-associated genotypes and premature birth, suggesting that during pregnancy, MBL-associated inflammation caused by higher MBL activity may contribute to earlier delivery. Furthermore, this finding might explain why so many individuals are MBL deficient in the general population (van de Geijn et al. 2008). Moreover, Swierzko et al. recently reported that the high serum MBL-conferring A/A genotypes in neonates are associated with prematurity. Consistent with that, low (deficiency) MBL levels were underrepresented among premature babies (Swierzko et al. 2009). It is very probable that although MBL is protective when a pathogen is to be eliminated by an inflammatory complement-dependent response, persistent activation may in some cases lead to an excessive response (Ameglio et al. 2007; Swierzko et al. 2009). Since, as mentioned, intrauterine infections are one of the main reasons for premature deliveries, it is possible that, however rarely, higher MBL levels might be disadvantageous. Indeed, these data add to the ever-increasing number of contexts in which high MBL seems to be harmful (Berger et al. 2007; Bilgin et al. 2008; Fiane et al. 2003; Hansen et al. 2006; Hellemann et al. 2007; Kilpatrick 2007; Schlapbach et al. 2007). On the other hand, there are numerous reports showing an association between MBL deficiency in mothers or babies with prematurity or prenatal/perinatal infections (Annells et al. 2004; De Benedetti et al. 2007; Bodamer et al. 2006; Frakking et al. 2007).

Type 1 diabetes, like rheumatoid arthritis, is an autoimmune disease and symptoms of rheumatoid arthritis sometimes improve during pregnancy. Increased concentration of MBL in the course of pregnancy might affect pregnancy outcome. Thus, probably, both total MBL deficiency and high level/activity during pregnancy complicated by diabetes might be dangerous for the fetus and/or mother.

We realize that the small numbers of patients in the study groups constitute a major limitation. It reflects, however, the very strict manner of their qualification. First, in the non-pregnant diabetic group all of the selected patients were nulliparous. In practice, in a hospital setting it is extremely difficult to obtain blood samples from women with diabetes who had never been pregnant before. It is only possible in centers providing special care for women with diabetes preparing for pregnancy. In 2007 the Society of Obstetricians and Gynecologists of Canada published guidelines for the management of diabetic pregnancy. It stated (just as in the St. Vincent declaration of 1989) that women with diabetes should be offered pre-conception counseling with a multidisciplinary team to optimize general health and glycemic control and to review the risks of congenital anomalies. Moreover, they emphasized that pregnancy in women with diabetes should be planned. Unfortunately, the awareness of such care in the diabetic population is still low and therefore most patients in our Diabetes Center of the RI PMMH (Lódź, Poland), are women who have already experienced miscarriage, lost a baby, or delivered a macrosomic baby or a baby with congenital malformations. Primigravidae were selected for our study group of pregnant diabetic women, which makes it clear why there were only 15 of them. Moreover, all of the patients were between 20-30 years of age. Again, in practice it is extremely difficult to find young women (mean age: 23.6 years) suffering from diabetes who are aware that they should report for pregnancy planning.

We believe that our study is the very first to show MBL/LP fluctuations in the course of pregnancy of diabetic primigravidae and provides a unique opportunity to observe these changes in a very strictly selected group of patients. Increasing levels of MBL (and other innate immunity factors) in the course of pregnancy may be necessary to compensate for reduced T-cell function during a state of reduced adaptive immunity. Higher levels of MBL and LP could indicate a shift from adaptive to innate immunity.

Acknowledgment: This study was supported by the Polish Ministry of Science and Higher Education Scientific Research Committee (grant no. 2P05E 024 30). The authors are very grateful Dr. David C. Kilpatrick for constructive criticism of the manuscript.

\section{REFERENCES}

Ameglio F, Vento G, Romagnoli C et al (2007) Association of MBL2 variants with early preterm delivery. Genet Med 9:136-137

Annells MF, Hart PH, Mullighan CG et al (2004) Interleukins $-1,-4,-6,-10$, tumor necrosis factor, transforming growth factor-beta, FAS, and mannose-binding protein $\mathrm{C}$ gene polymorphisms in Australian women: Risk of preterm birth. Am J Obstet Gynecol 191: 2056-2067

Beagley KW, Gockel CM (2003) Regulation of innate and adaptive immunity by the female sex hormones oestradiol and progesterone. FEMS Immunol Med Microbiol 38:13-22

Berger SP, Roos A, Mallat MJ et al (2007) Low pretransplant mannose-binding lectin levels predict superior patient and graft survival after simultaneous pancreas-kidney transplantation. J Am Soc Nephrol 18:2416-2422

Bilgin YM, Brand A, Berger SP et al (2008) Mannose-binding lectin is involved in multiple organ dysfunction syndrome after 
cardiac surgery: effects of blood transfusions. Transfusion 48:601-608

Bodamer OA, Mitterer G, Maurer W et al (2006) Evidence of a correlation between mannose-binding lectin (MBL2) gene polymorphisms and preterm birth. Genet Med 8:518-524

Bouwman LH, Eerligh P, Terpstra OT et al (2005) Elevated levels of mannose-binding lectin at clinical manifestation of type 1 diabetes in juveniles. Diabetes 54:3002-3006

Cedzynski M, Szemraj J, Swierzko AS et al (2004) Mannan-binding lectin insufficiency in children with recurrent infections of the respiratory system. Clin Exp Immunol 136:304-311

Chomczynski P (1993) A reagent for single-step simultaneous isolation of RNA, DNA and proteins from cell and tissue samples. Biotechniques 3:532-537

Dahl MR, Thiel S, Matsushita M et al (2001) MASP-3 and its association with distinct complexes of the mannan-binding lectin complement activation pathway. Immunity 1:127-135

De Benedetti F, Auriti C, D’Urbano LE et al (2007) Low serum levels of mannose binding lectin are a risk factor for neonatal sepsis. Pediatr Res 61:325-328

Fiane AE, Videm V, Lingaas PS et al (2003) Mechanism of complement activation and its role in the inflammatory response after thoracoabdominal aortic aneurysm repair. Circulation 108:849-856

Frakking FN, Brouwer N, van Eijkelenburg NK et al (2007) Low mannose-binding lectin (MBL) levels in neonates with pneumonia and sepsis. Clin Exp Immunol 150:255-262

Hansen TK, Gall M-A, Tarnow L et al (2006) Mannose-binding lectin and mortality in type 2 diabetes. Arch Intern Med 166:2007-2013

Hansen TK, Tarnow L, Thiel S et al (2004) Association between mannose-binding lectin and vascular complications in type 1 diabetes Diabetes 53:1570-1576

Hansen TK, Thiel S, Knudsen ST et al (2003a) Elevated levels of mannan-binding lectin in patients with type 1 diabetes. J Clin Endocrinol Metab 88:4857-4861

Hansen TK, Thiel S, Wouters PJ et al (2003b) Intensive insulin therapy exerts antiinflammatory effects in critically ill patients and counteracts the adverse effect of low mannose-binding lectin levels. J Clin Endocrinol Metab 88:1082-1088

Hellemann D, Larsson A, Madsen HO et al (2007) Heterozygosity of mannose-binding lectin (MBL2) genotypes predicts advantage (heterosis) in relation to fatal outcome in intensive care patients. Hum Mol Genet 16:3071-3080

Hovind P, Hansen TK, Tarnow L et al (2005) Mannose-binding lectin as a predictor of microalbuminuria in type 1 diabetes: an inception cohort study. Diabetes 54:1523-1527

Husby S, Herskind AM, Jensenius JC et al (2002) Heritability estimates for the constitutional levels of the collectins mannan-binding lectin and lung surfactant protein D. A study of unselected like-sexed mono- and dizygotic twins at the age of 6-9 years. Immunology 106:389-394

Kaushic C, Ashkar AA, Reid LA et al (2003) Progesterone increases susceptibility and decreases immune responses to genital herpes infection. J Virol 77:4558-4565
Kilpatrick DC (2000) Mannan-binding lectin concentration during normal human pregnancy. Hum Reprod 15:941-943

Kilpatrick DC (2007) Clinical significance of mannan-binding lectin and L-ficolin. In: Kilpatrick D (ed) Collagen-related lectins in innate immunity. Research Signpost, Trivandrum, pp $57-84$

Koch CA, Platt JL (2003) Natural mechanisms for evading graft rejection: the fetus as an allograft. Springer Semin Immunopathol 25:95-117

Kruse C, Rosgaard A, Steffensen R et al (2002) Low serum level of mannan-binding lectin is a determinant for pregnancy outcome in women with recurrent spontaneous abortion. Am J Obstet Gynecol 187:1313-1320

Madsen HO, Garred P, Thiel S et al (1995) Interplay between promoter and structural gene variants control basal serum level of MBL. J Immunol 155:3013-3020

Matsushita M, Fujita T (1992) Activation of the classical complement pathway by mannose-binding protein in association with a novel C1s-like serine protease. J Exp Med 6:1497-1502

Megia A, Gallart L, Fernández-Real JM et al (2004) Mannosebinding lectin gene polymorphisms are associated with gestational diabetes mellitus. J Clin Endocrinol Metab 89:5081-5087

Pertynska M, Tchorzewski H, Cedzynski M et al (2001) Levels of mannose binding lectin in early pregnancy complicated by diabetes mellitus type 1 - preliminary report. Ginekol Pol 72:1267-1271

Petersen SV, Thiel S, Jensen L et al (2001) An assay for the mannan-binding lectin pathway of complement activation. J Immunol Methods 257:107-116

Raghupathy R (1997) Th1-type immunity is incompatible with successful pregnancy. Immunol Today 18:478-482

Schlapbach LJ, Aebi C, Otth M et al (2007) Serum levels of mannose-binding lectin and the risk of fever in neutropenic pediatric cancer patients. Pediatr Blood Cancer 49:11-16

Sorensen CM, Hansen TK, Steffensen R et al (2006) Hormonal regulation of mannan-binding lectin synthesis in hepatocytes. Clin Exp Immunol 145:173-182

Swierzko A, Atkinson AP, Cedzynski M et al (2009) Two factors of the lectin pathway of complement, L-ficolin and mannan-binding lectin, and their associations with prematurity, low birthweight and infections in a large cohort of Polish neonates. Mol Immunol 46:551-558

Szekeres-Bartho J, Par G, Szereday L et al (1997) Progesterone and non-specific immunologic mechanisms in pregnancy. Am J Reprod Immunol 38:176-182

Thiel S, Møller-Kristensen M, Jensen L et al (2002) Assays for the functional activity of the mannan-binding lectin pathway of complement activation. Immunobiology 205:446-454

Thiel S, Vorup-Jensen T, Stover CM et al (1997) A second serine protease associated with mannan-binding lectin that activates complement. Nature 386:506-510

Tsutsumi A, Ikegami H, Takahashi R et al (2003) Mannose binding lectin gene polymorphism in patients with type I diabetes. Hum Immunol 64:621-624 
Turner MW (1996) The lectin pathway of complement activation. Res Immunol 147:110-115

Turner MW, Hamvas RM (2000) Mannose-binding lectin: structure, function, genetics and disease associations. Rev Immunogenet 3:305-322 van de Geijn FE, Dolhain RJ, van Rijs W et al (2008) Mannose-binding lectin genotypes are associated with shorter gestational age. An evolutionary advantage of low MBL production genotypes? Mol Immunol 45:1514-1518

van de Geijn FE, Roos A, de Man YA et al (2007) Mannose-binding lectin levels during pregnancy: a longitudinal study. Hum Reprod 22:362-371 